\title{
Les évolutions passées et futures du climat de la Nouvelle-Calédonie
}

\author{
Virgil Cavarero(1), Alexandre Peltier(1), Xavier Aubail(1), \\ Anne Leroy(1), Brigitte Dubuisson(2), Sylvie Jourdain(2), \\ Alexandre Ganachaud(3), Anne-Laure Gibelin(2), Jérôme Lefèvre(3), \\ Christophe Menkes(3) et Matthieu Lengaigne ${ }^{(4)}$ \\ (1) Météo-France Nouvelle-Calédonie \\ 5, rue Vincent Auriol, Faubourg Blanchot, BP 151 - 98845 Nouméa Cedex \\ (2) Météo-France, Direction de la climatologie, Toulouse \\ (3) Institut de recherche pour le développement (IRD), Nouméa \\ (4) Laboratoire d'océanographie et du climat, expérimentations \\ et approches numériques (LOCEAN), Paris
}

\section{Résumé}

Les longues séries d'observations de températures et de précipitations de la fin $d u x^{e}$ siècle en NouvelleCalédonie ont été homogénéisées puis analysées. Les moyennes annuelles des températures minimales et maximales ont augmenté respectivement de 0,3 et $0,2{ }^{\circ} \mathrm{C}$ par décennie sur la période 1970-2009. Les effets futurs du changement climatique en Nouvelle-Calédonie ont été étudiés en effectuant une descente d'échelle par la méthode quantile-quantile à partir de simulations numériques réalisées pour le quatrième rapport du GIEC. Selon les scénarios d'émissions de gaz à effet de serre, l'augmentation des températures minimales et maximales se situerait entre $+1,5$ et $+2,7^{\circ} \mathrm{C}$ en un siècle, relativement à la période 1971-1999. Il apparaît également que les conditions de température du futur entraîneraient une augmentation de la fréquence des années météorologiquement favorables aux épidémies de dengue. Quant aux cumuls annuels de précipitations, aucune tendance significative n'apparaît, ni dans les observations, ni dans les projections. a Nouvelle-Calédonie présente $a$ priori une vulnérabilité importante vis-à-vis du changement climatique, en raison notamment de la constante augmentation des pressions sur ses ressources en eau, de l'importance de sa biodiversité et de son récif corallien (sensible à la température de l'eau), sans oublier les risques que représentent les feux de brousse et les épidémies de dengue.

Le changement climatique peut y être mis en évidence sur la fin du $\mathrm{XX}^{\mathrm{e}}$ siècle grâce à de longues séries de mesures de températures et de précipitations dont on a pris soin, au préalable, de s'assurer de la qualité par une méthode d'homogénéisation, au pas de temps mensuel puis quotidien.

La question se pose ensuite de l'évolution des principales variables climatiques au cours du XXI ${ }^{\mathrm{e}}$ siècle. Le dernier rapport du Groupe d'experts intergouvernemental sur l'évolution du climat (GIEC), paru en 2007, a fourni des projections climatiques de 2000 à 2100. Notre apport a consisté à adapter les résultats des modèles en prenant en compte les particularités locales, par la méthode quantile-quantile.

Nous disposons ainsi de séries quotidiennes corrigées qui permettent de dégager les tendances éventuelles de précipitations et de températures de la période actuelle au milieu du XXI ${ }^{\mathrm{e}}$ siècle (entre 2046 et 2064) et de la période actuelle à la fin du XXI ${ }^{\mathrm{e}}$ siècle (entre
2081 et 2099). Un indice particulier sera analysé ici, en lien avec les risques d'épidémie de dengue : le nombre de jours pendant lesquels la température maximale dépasse le seuil de $32^{\circ} \mathrm{C}$.

\section{Le climat de la Nouvelle-Calédonie a changé}

\section{L'homogénéité des données : un examen primordial}

Pour mettre en évidence les évolutions passées du climat de leur territoire, les météorologues calédoniens disposent de longues séries de températures et de précipitations, mesurées par un réseau de stations (désormais automatiques) et par des observateurs bénévoles.

Bien que des contrôles réguliers rendent les données très fiables, le remplacement d'un capteur, le déplacement d'une station de quelques centaines de mètres ou une modification brutale de son environnement peuvent induire des discontinuités non climatiques nettes dans la série de données. Or, l'amplitude de ces biais est souvent du même ordre de grandeur que les variations climatiques que l'on cherche à mettre en évidence. La détection et la correction de ces « ruptures » d'homogénéité dans les séries de données climatiques sont 


\section{Abstract}

\section{Past and future changes of New-Caledonia's climate}

Homogenised series of temperature and precipitation data for the late $20^{\text {th }}$ century in New-Caledonia are presented. Annual mean minimum and maximum temperatures have increased by 0.3 and $0.2^{\circ} \mathrm{C}$ per decade respectively. A quantile-quantile downscaling method has been performed on IPCC AR4 numerical simulations in order to study the impact of climate change on NewCaledonia in the future. The minimum and maximum temperatures would increase by between 1.5 and $2.7^{\circ} \mathrm{C}$ by the end of the $21^{\text {st }}$ century, relative to the 1971-1999 period, depending on greenhouse gases emission scenarios. It also appears that the temperature of the future would support an increase in the frequency of the years that climatically favour outbreaks of dengue fever. No significant trend appears in observations of total annual precipitation, or in projections. donc des préliminaires incontournables à toute étude sur l'évolution des conditions climatiques locales (Moisselin et al., 2002 ; Aguilar et al., 2003).

\section{Données mensuelles et évolutions générales du climat}

Le climat calédonien s'est-il humidifié, s'est-il réchauffé ? Pour répondre à ces questions, relatives aux évolutions moyennes du climat, on a recours aux séries de données mensuelles.

Afin de supprimer les ruptures d'homogénéité de ces séries, nous employons la méthode d'homogénéisation appelée PRODIGE(1) (Caussinus et Mestre, 2004). Par rapport aux autres outils disponibles, PRODIGE présente l'avantage de ne pas recourir à une série de référence, prétendument homogène, pour permettre la détection et la correction de ruptures d'une série, et de ne pas nécessiter la connaissance au préalable du nombre de ruptures significatives à traiter. Comme la méthode est fondée sur la comparaison de séries d'un même voisinage climatique, son utilisation requiert néanmoins un nombre suffisant de séries bien corrélées entre elles.

Ainsi 28 séries homogénéisées de cumuls mensuels de précipitations ontelles été obtenues sur la période 19612008 et 16 séries de moyennes mensuelles de températures minimales et de températures maximales, sur la période 1970-2009. La plupart des séries de températures présentent au moins une rupture, en raison notamment du remplacement des abris météorologiques. Quant aux séries de précipitations, 9 stations sur 28 présentent des ruptures sans équivoque. Toutefois, l'origine d'une seule rupture a pu être identifiée grâce aux annales de maintenance.

Afin d'analyser les évolutions générales du climat calédonien, il est d'usage de vérifier si les différentes séries présentent une tendance, à l'aide du test statistique, réputé robuste, de Mann-Kendall. Cet examen est complété par l'estimation de la tendance linéaire grâce à la méthode des moindres carrés. Le niveau de confiance associé aux différents tests est fixé à $95 \%$.

Figure 1 - Moyennes annuelles de la série homogénéisée des moyennes mensuelles des températures minimales mesurées à Nouméa de 1970 à 2009. L'amplitude des anomalies négatives (respectivement positives), qui correspondent à des valeurs inférieures (respectivement supérieures) à la moyenne annuelle calculée sur toute la période, est représentée en bleu (resp. rouge). La droite issue de la régression linéaire, par la méthode des moindres carrés, est matérialisée par la ligne pointillée.

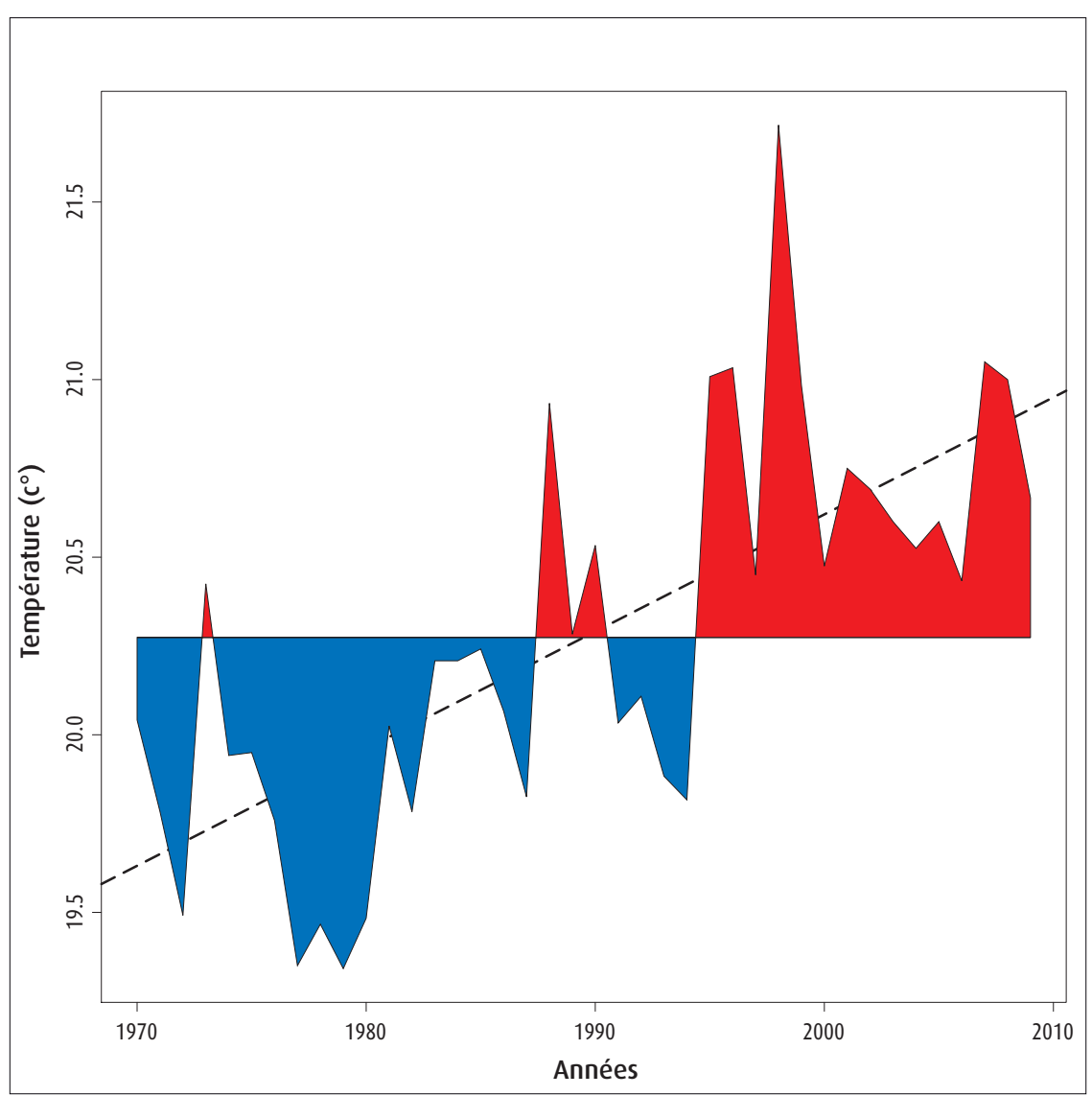


Les calculs mettent en évidence qu'au cours des 40 dernières années les moyennes annuelles des températures minimales et maximales quotidiennes ont augmenté respectivement de $0,3{ }^{\circ} \mathrm{C}$ et $0,2^{\circ} \mathrm{C}$ par décennie, avec une incertitude dans les deux cas de $\pm 0,1^{\circ} \mathrm{C}$ par décennie, comme l'illustre la figure 1 pour Nouméa. Le test de Mann-Kendall tend à indiquer que ces tendances sont significatives au seuil de confiance $95 \%$, c'est-à-dire qu'elles se distinguent de la variabilité d'une année sur l'autre. De plus, on ne peut raisonnablement pas affirmer qu'une partie du territoire s'est réchauffée plus vite que les autres.

Enfin, il est important de noter que les tendances globales sur la période 19702009 sont supérieures aux amplitudes des ruptures pouvant subsister dans les séries, qui sont au maximum de l'ordre de $0,2^{\circ} \mathrm{C}$, d'après PRODIGE.

Pour les précipitations, l'analyse est moins concluante. Premièrement, leur forte variabilité spatiotemporelle dégrade sensiblement les performances de PRODIGE. On estime que dans les séries homogénéisées subsisteraient des ruptures pouvant atteindre entre 15 et $20 \%$ des normales annuelles. Deuxièmement, les séries sont relativement courtes eu égard à leur variabilité interannuelle élevée, ce qui rend difficile la détection d'une tendance climatique. Bien qu'aucune tendance statistiquement significative ne soit décelable, nous ne pouvons donc pas conclure de manière définitive quant à l'évolution des cumuls annuels de précipitations entre 1961 et 2008. Seul le régime des pluies observées entre juin et août se caractérise par une évolution temporelle sensible. Pour une grande majorité des postes de la côte est (7 sur 10), ainsi qu'à Lifou (Ouanaham), les quantités de précipitations de ce trimestre ont significativement diminué. Par exemple, on remarque qu'à Ponérihouen (figure 2) la période antérieure à 1980 est dominée par des hivers très pluvieux alors que, par la suite, le ciel a plutôt été avare en précipitations. Pour ces 8 postes, la tendance avoisine les $-45 \%$. Toutefois, l'incertitude associée à cette estimation est plutôt élevée, puisqu'on évalue qu'il y a $95 \%$ de chance pour que ce taux se situe entre -85 et $-5 \%$. Cette tendance, qui équivaut à $5 \%$ du cumul annuel, est inférieure à l'amplitude minimale des ruptures détectables par PRODIGE. Pour 19 des 20 autres postes du territoire, la tendance est également négative mais elle n'est pas significative sur le plan statistique.

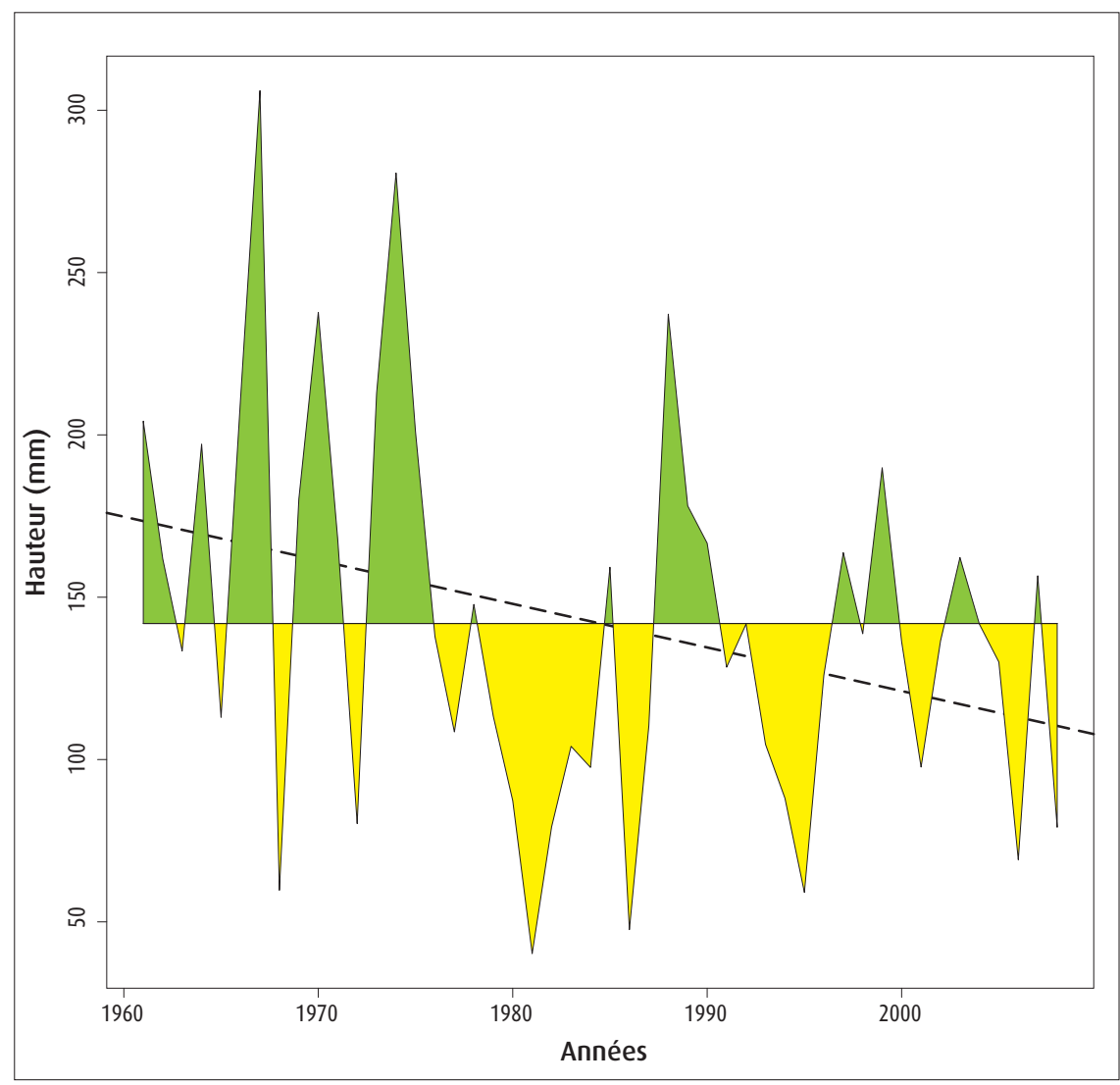

Figure 2 - Moyennes pour le trimestre juin-juillet-août de la série homogénéisée des cumuls mensuels des précipitations mesurées à Ponérihouen de 1961 à 2008. L'amplitude des anomalies négatives (respectivement positives), qui correspondent à des valeurs inférieures (resp. supérieures) à la moyenne calculée sur toute la période, est représentée en jaune (resp. vert). La droite issue de la régression linéaire, par la méthode des moindres carrés, est matérialisée par la ligne pointillée.

\section{Données quotidiennes et évolution des extrêmes}

Qu'en est-il de l'évolution des événements météorologiques notables, comme les jours pluvieux ou les jours de fortes chaleurs ? La réponse se trouve dans l'examen des données quotidiennes.

Pour détecter les ruptures au sein des séries quotidiennes, il est judicieux de reprendre les résultats trouvés par PRODIGE au pas de temps mensuel (Mestre, 2007). La correction des données quotidiennes est, quant à elle, une opération des plus ardues car, contrairement au pas de temps mensuel, on ne peut pas faire l'hypothèse simplificatrice qu'un changement dans les conditions de mesure introduit un biais constant dans la série de données. Par exemple, les effets du déplacement d'un abri météorologique sur la mesure de la température peuvent varier suivant les conditions d'ensoleillement et la température.

Pour les données pluviométriques, la démarche la plus raisonnable ne consiste pas à chercher à corriger quotidiennement les données mais à utiliser uniquement les séries pour lesquelles PRODIGE n'a détecté aucune rupture. Ces données existent en nombre suffisant pour obtenir des résultats concluants : 19 séries sur les 28 contrôlées initialement. En revanche, PRODIGE a mis en évidence que la plupart des données thermométriques présentent au moins une rupture significative. Il est donc nécessaire de corriger ces dernières. Pour ce faire, on met en œuvre une simple interpolation temporelle des coefficients mensuels de correction (Vincent et al., 2002).

Les paramètres quotidiens sont examinés à travers une sélection d'indices définis par le groupe d'experts ETCCDI (Expert Team on Climate Change Detection and Indices). Les calculs sont réalisés à l'aide du logiciel RClimDex (Zhang et Feng, 2004). Un exemple d'indice lié à la température sera évoqué plus loin au paragraphe « Évolution des températures maximales par rapport au seuil de $32{ }^{\circ} \mathrm{C} »$.

On s'intéresse d'abord au nombre annuel de jours pluvieux pour lesquels le cumul quotidien a dépassé $1 \mathrm{~mm}$ 


\section{Climat de la Nouvelle-Calédonie}

Située dans le Pacifique sud-ouest, la Nouvelle-Calédonie est un archipel composé d'une île principale, appelée Grande Terre, et d'îles secondaires : l'île des Pins, au sud, et les îles Loyauté à l'est (Maré, Tiga, Lifou et Ouvéa). La Grande Terre est une île à la forme effilée qui s'étend sur plus de 400 km en longueur, pour seulement 50 à 70 km en largeur. Bien qu'à l'échelle climatique ce soit un petit pays, ne vous y méprenez pas, la Nouvelle-Calédonie est bien plus grande que tous les territoires français ultramarins, la Guyane exceptée. L'île de Lifou, par exemple, fait $100 \mathrm{~km}^{2}$ de plus que la Martinique !

Au centre de la Grande Terre, le long d'un axe sud-est/nord-ouest, s'étend une chaîne montagneuse dont les plus hauts sommets culminent à plus de $1600 \mathrm{~m}$ d'altitude. Les autres îles sont relativement plates et proches du niveau de la mer.

Situé sous le tropique du Capricorne, l'archipel subit les influences tropicales et tempérées plus ou moins fortement selon les saisons (Maitrepierre et Caudmont, 2007). On distingue deux saisons principales :

- La saison chaude est centrée sur le premier trimestre. L'influence tropicale est prédominante et le temps est rythmé par la position de la Zone de convergence du Pacifique sud (ZCPS), ainsi que par les trajectoires des dépressions tropicales. Les précipitations sont abondantes et les températures moyennes sont élevées bien que les extrêmes soient limités par l'influence maritime et l'alizé. -En saison fraîche, de juin à septembre, la ZCPS se décale vers le nord-est. Les perturbations des régions tempérées remontant vers le nord se manifestent, en Nouvelle-Calédonie, par des précipitations et, parfois, par des " coups d'ouest ». Ces épisodes perturbés ponctuent un temps généralement sec et frais avec des températures minimales relativement basses en certaines régions.

La transition entre ces deux saisons n'est pas toujours évidente à distinguer :

- La saison sèche, d'août à novembre, se trouve à cheval entre la saison fraîche et la saison chaude. Cette partie de l'année se caractérise par des précipitations très faibles et par des températures de plus en plus élevées la journée. Les feux de forêt se propagent facilement sur une végétation déshydratée, sous l'action d'un alizé renforcé par les brises. Le retour des précipitations est donc très attendu, mais il peut être dramatiquement retardé pendant les épisodes El Niño.

-En fin de saison chaude/début de saison fraîche, la température de l'eau de mer est encore chaude et elle peut favoriser la formation d'épisodes pluvio-orageux importants, voire de dépressions subtropicales.

ENSO (EI Niño Southern Oscillation) est la principale source de variabilité interannuelle des précipitations et des températures localement observées en Nouvelle-Calédonie (Leroy, 2006) :

- Les précipitations sont fortement affectées par ENSO, surtout en saison chaude et particulièrement dans le nord de la Grande Terre et sur les îles Loyauté. Les périodes El Niño sont caractérisées par un risque accru de sécheresse et les périodes La Niña, par un risque accru de fortes pluies.

- Les températures minimales ont tendance à être anormalement basses en périodes El Niño ; c'est l'opposé en périodes La Niña. - La relation entre ENSO et les températures maximales est moins marquée. Elle varie suivant la saison et la région considérée. L'influence d'ENSO sur les températures maximales est faible en saison chaude.

(voir l'exemple de la figure 3). Le test de Mann-Kendall met en évidence que le nombre de jours de pluie se serait accru pour 7 des 16 séries examinées, au cours des 5 dernières décennies. Pour ces séries, l'estimation de la tendance oscille entre +10 et $+40 \%$ de la moyenne. Aucune cohérence spatiale des résultats n'apparaît. Pour les autres postes, l'évolution est aussi à la hausse, à deux exceptions près, mais la tendance n'est pas significative au seuil de confiance fixé $(95 \%)$.

L'autre évolution notable est celle des cumuls des fortes précipitations de l'hiver austral, définis comme la somme des $5 \%$ des pluies quotidiennes les plus élevées entre juin et août. On remarque que cette quantité a tendance à décroître pour les postes de la côte est ainsi qu'à Lifou (7 postes au total), ce qui corrobore bien la diminution des précipitations totales signalée au paragraphe «Données mensuelles et évolutions générales du climat ». De surcroît, pour 5 de ces stations, le nombre de jours au cours desquels les pluies ont dépassé $20 \mathrm{~mm}$ a diminué. Il semble donc que les épisodes de pluies substantielles, entre juin et août, ont diminué en intensité et en nombre sur la côte est et à
Figure 3 - Nombre de jours par an pour lesquels le cumul quotidien de précipitations a dépassé $1 \mathrm{~mm}$, pour la station de Nouméa, entre 1961 et 2008. Mêmes conventions que pour la figure 2.

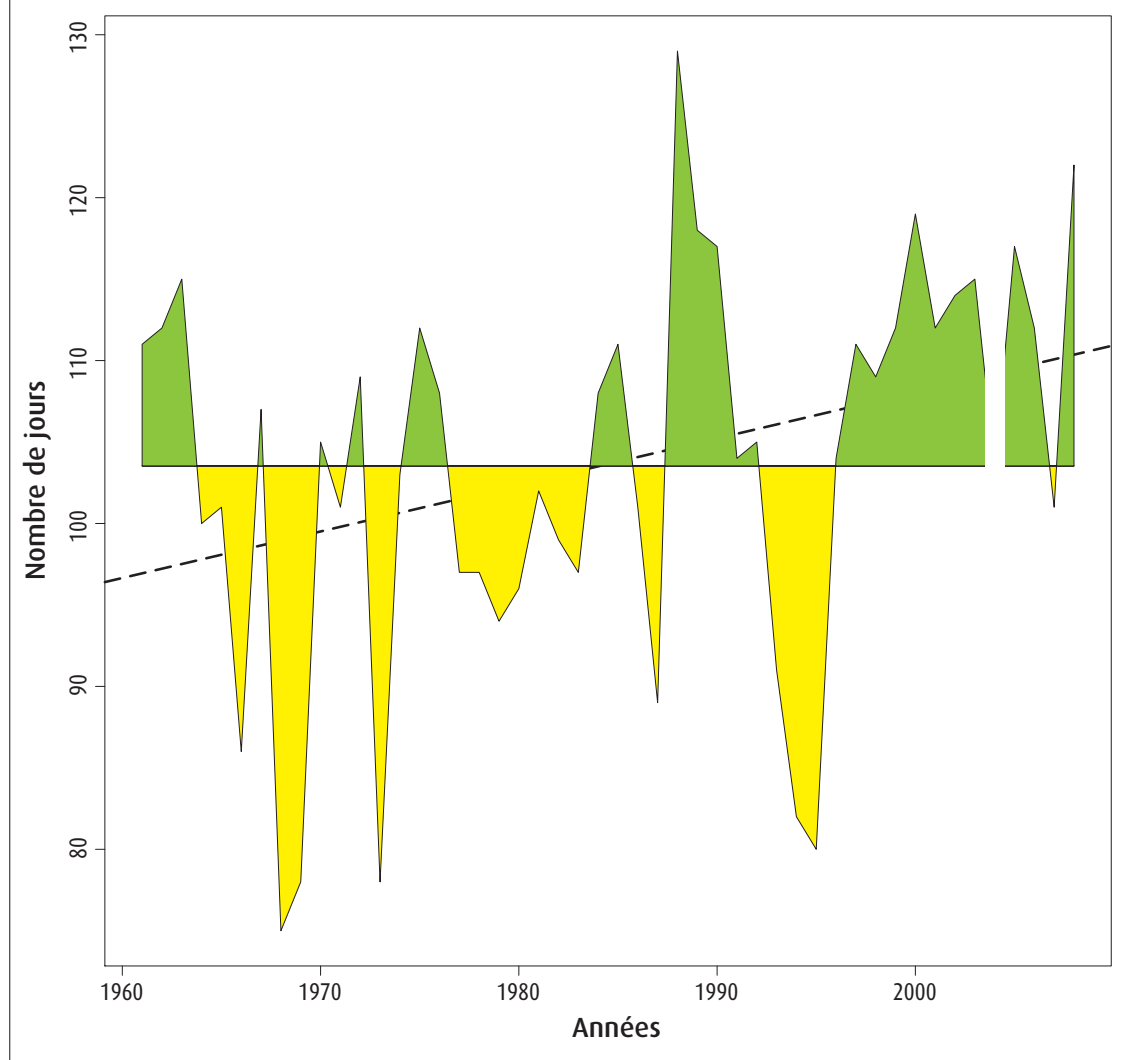


Lifou. Pour les autres postes, à une exception près, on peut identifier une diminution mais elle n'est pas significative. Encore une fois, bien qu'ils soient significatifs au sens statistique, ces résultats sont sujets à caution en raison de l'incertitude sur la détection des ruptures des séries pluviométriques.

\section{Le climat de la Nouvelle-Calédonie va continuer à changer}

Voyons maintenant comment pourrait évoluer le climat calédonien au XXI ${ }^{\mathrm{e}}$ siècle. On s'intéresse à 5 postes, représentés sur la figure 4, dont la répartition géographique prend en compte la variabilité du climat entre la Grande Terre et les îles Loyauté, mais aussi entre la côte ouest et la côte est.

\section{Simulations des modèles du GIEC}

\section{Les scénarios climatiques utilisés}

L'évolution du climat étant influencée par les activités humaines, il est nécessaire, pour simuler le climat jusqu'en 2100 , de faire des hypothèses sur la

\begin{tabular}{l|c|l|l}
\hline Modèle & Version & \multicolumn{1}{c}{ Laboratoire } & \multicolumn{1}{c}{ Pays } \\
\hline CSIR0 Mk3.5d & 2005 & CSIRO & Australie \\
\hline GFDL-CM2.0 & 2004 & U.S. Department of Commerce/NOAA/GFDL & États-Unis \\
\hline GFDL-CM2.1 & 2004 & U.S. Department of Commerce/NOAA/GFDL & États-Unis \\
\hline ECHO-G & 1999 & MIUB/KMA & Allemagne, Corée du Sud \\
\hline ECHAM5/MPI-0M & 2004 & MPI & Allemagne \\
\hline MRI-CGCM2.3.2a & 2001 & MRI & Japon \\
\hline Tableau 1 - Caractéristiques des six modèles du GIEC utilisés. &
\end{tabular}

croissance de la population mondiale et sur la consommation d'énergies fossiles. Ainsi les simulations réalisées dans le cadre du GIEC pour le XXI ${ }^{\mathrm{e}}$ siècle suivent-elles des scénarios dits « SRES » (Special Report on Emissions Scenarios). Deux scénarios sont étudiés ici : SRES A2, pour lequel les émissions de $\mathrm{CO}_{2}$ continuent de croître jusqu'en 2100, et SRES B1, pour lequel ces émissions décroissent à partir de 2050.

On peut se demander si ces scénarios sont réalistes. En effet, entre 2000 et 2011 , les émissions de $\mathrm{CO}_{2}$ ont dépassé ce qui avait été imaginé par le scénario A2, pourtant le plus pessimiste (voir les données récentes sur http://www.universcience.fr/climobs/). À l'inverse, les scénarios basés sur une limitation des émissions dans un avenir proche sont cohérents avec la limitation des stocks d'énergie non renouvelables. Le large éventail d'émissions proposé par les scénarios SRES, comme par ceux du prochain rapport du GIEC (Representative Concentration Pathways, ou RCP), permet de prendre en compte les incertitudes sur les concentrations en gaz à effet de serre, incertitudes qui restent fortes car elles dépendent de choix sociétaux et industriels.

Les simulations du $\mathrm{XX}^{\mathrm{e}}$ siècle suivant le scénario 《 $20 \mathrm{c} 3 \mathrm{~m}$ 》 fournissent des séries quotidiennes, entre 1961 et 2000, qui incluent des concentrations de gaz à effet de serre augmentant, conformément aux observations. Ces séries permettent de contrôler l'accord entre les simulations et les observations et elles servent de référence pour évaluer l'évolution future du climat.

\section{Choix des modèles du GIEC}

La présente étude se fonde sur les simulations réalisées pour le quatrième rapport du GIEC. Six modèles (listés au tableau 1) ont été sélectionnés pour leur représentation raisonnable des phénomènes influençant le climat de la Nouvelle-Calédonie, à savoir la Zone de convergence du Pacifique sud (ZCPS) et El Niño Oscillation australe (ENSO), selon les trois critères suivants :

- qualité de la représentation de l'état moyen (latitude et pente) de la ZCPS (Brown et al., $2011 \mathrm{a}$; Vincent, 2008);

- qualité de la représentation de l'ENSO (Lin, 2007 ; Van Oldenborgh et al., 2005 ; Leloup et al., 2008 ; Guilyardi et al., 2009);

Figure 4 - Carte de NouvelleCalédonie situant les stations utilisées : Nouméa, Bourail (ou Nessadiou pour les températures), Ouanaham, Koumac et Touho (ou Poindimié pour les températures). En rouge sont représentés les points de grille du modèle australien du CSIRO (Commonwealth Scientific and Industrial Research Organisation), doté d'une maille de $200 \times 200 \mathrm{~km}$. 
- variabilité de la ZCPS associée à l'ENSO, et capacité à produire des modes symétriques et asymétriques (Vincent et al., 2009).

\section{La méthode quantile-quantile}

Les modèles développés par les climatologues pour étudier le changement climatique sont conçus pour des simulations longues et possèdent, en contrepartie, une résolution faible et une géographie simplifiée (dans la plupart des modèles, il n'y a pas de point de terre au niveau de la Nouvelle-Calédonie). Cela s'avère suffisant pour le climat global ou à l'échelle des continents mais pas pour le climat d'une petite région. Il est donc nécessaire de réaliser une " descente d'échelle » pour adapter les résultats des modèles en tenant compte des particularités locales observées par le passé. Les méthodes statistiques de régionalisation, comparées aux méthodes dynamiques, présentent l'avantage d'être moins coûteuses en temps de calcul. Parmi les méthodes statistiques, la méthode de correction quantile-quantile, qui a été choisie, est adaptée pour une première approche. Cette méthode est un peu plus élaborée que celle qui consiste à corriger seulement le biais moyen et elle est assez robuste comparée aux méthodes de type « régression » (Déqué, 2007). Non seulement la méthode quantilequantile possède l'avantage de corriger les biais du modèle mais elle convient également à l'étude des extrêmes, pour évaluer les fréquences de dépassement de seuils en certains points, car la correction à apporter à la valeur simulée tient compte de la fréquence d'occurrence de l'événement considéré.

Les quantiles utilisés dans cette étude sont des centiles. Pour mémoire, les centiles d'un jeu de données sont les 99 valeurs qui divisent en 100 parts égales les données triées par ordre croissant. Chaque intervalle représente donc $1 \%$ de l'échantillon de population concerné.

Dans un premier temps, la méthode quantile-quantile consiste à déterminer une relation simple, sur une période passée, entre les centiles calculés à partir des observations d'une station donnée et les centiles calculés au point de grille du modèle le plus proche de la station. Dans un second temps, les centiles du modèle sur la simulation du climat futur (SRES B1 ou A2) sont corrigés en utilisant la relation calculée au préalable, et on peut ainsi reconstituer des séries sans biais, en fonction de la station utilisée, en faisant toutefois l'hypothèse de la

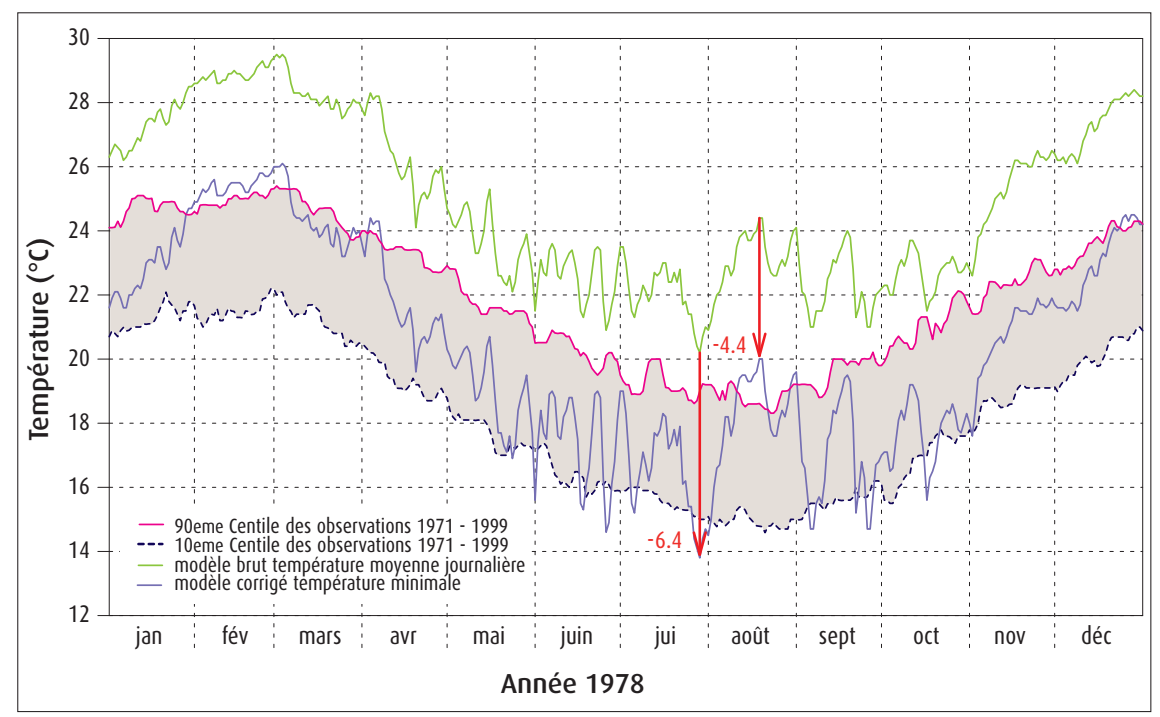

A Figure 5 - Pour l'année 1978, série brute et série corrigée de température minimale du modèle du CSIR0 pour Nouméa. En rouge, valeurs des corrections du 28 juillet et du 18 août 1978. La zone colorée correspond au cycle annuel des dixièmes et quatre-vingt dixièmes centiles des températures minimales observées, calculés sur la période de référence 1971-1999.

stabilité temporelle de la relation quantilequantile. Cependant, les incertitudes propres à la méthode quantile-quantile sont certainement négligeables par rapport à celles liées aux scénarios, aux simulations et à l'hypothèse de stabilité de la relation quantile-quantile.

La figure 5 illustre la méthode de correction. C'est justement parce que les corrections sont différentes pour chaque valeur (ici, plus la température modélisée est faible, plus la correction est importante en valeur absolue) que la correction a pour conséquence de ramener la série du modèle entre les dixièmes et quatre-vingt dixièmes centiles des observations et de faire en sorte que l'amplitude des fluctuations quotidiennes de température soit conforme aux observations, contrairement à la méthode des biais qui ne fait coïncider que les moyennes.

\section{Climat prévu en 2046-2064 et en 2081-2099}

\section{Évolution du cycle saisonnier}

Les modèles reproduisent correctement le cycle des températures et des précipitations (par exemple, à Nouméa sur la figure 6). Pour les températures, entre les observations ou la simulation de contrôle et les projections, la même augmentation s'applique à chaque mois, donc il n'y a pas de changement notable de l'amplitude ou de la phase saisonnière. À Nouméa, la saison chaude (au sens large) dure 6 mois, de novembre à avril. Sur la période 19711999, elle est caractérisée par des températures minimales moyennes supérieures à $20^{\circ} \mathrm{C}$ et par des températures maximales moyennes supérieures à $26{ }^{\circ} \mathrm{C}$. Entre 2081 et 2099, la période pendant laquelle les températures

จFigure 6 - Évolution du climat moyen de la station de Nouméa entre 1971-1999 (observations, simulations corrigées 20c3m) et 2081-2099 (simulations corrigées SRES B1 et SRES A2). Les précipitations mensuelles sont représentées par les barres grises, les températures minimales par les courbes bleues et les températures maximales par les courbes rouges.

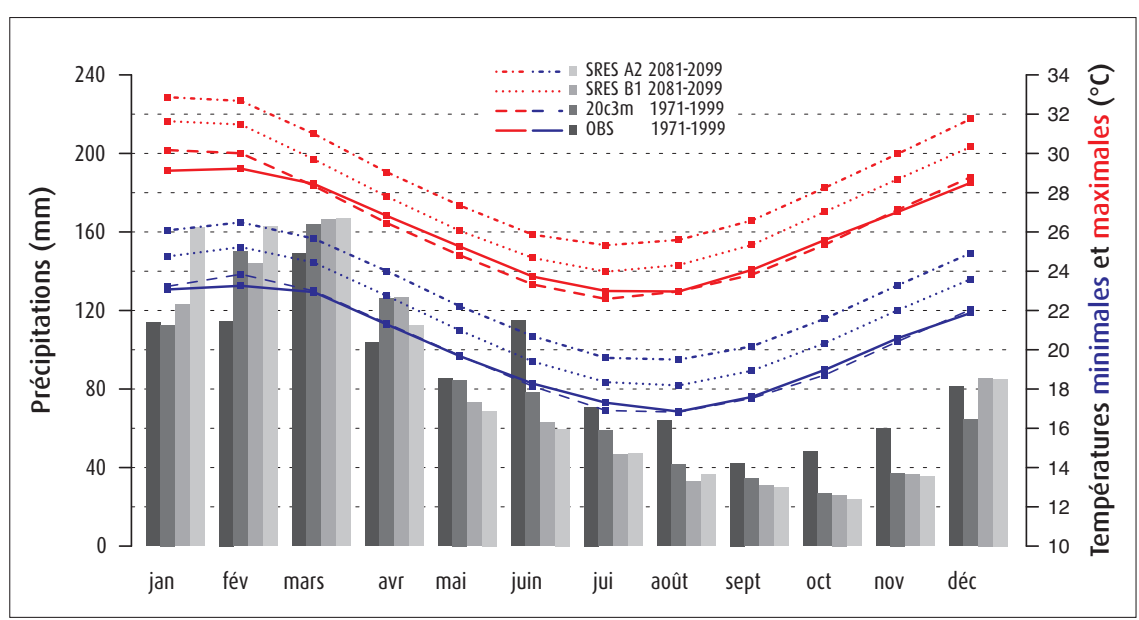


minimales et maximales moyennes dépasseraient ces seuils durerait 8 mois (d'octobre à mai), d'après le scénario SRES B1, et elle durerait 9 mois (de septembre à mai), selon le scénario SRES A2. Dès le milieu du XXI ${ }^{\mathrm{e}}$ siècle, la saison chaude serait allongée d'un mois et demi.

\section{Évolution des moyennes annuelles de températures}

Pour les 5 stations, l'augmentation des températures minimales et maximales moyennées sur la période se situerait entre 0,8 et $1,2{ }^{\circ} \mathrm{C}$, de la période actuelle à la période 2046-2064, selon le scénario d'émissions limitées (SRES B1), et entre 1,1 et $1,7^{\circ} \mathrm{C}$, selon le scénario d'émissions persistantes (SRES A2). Comme 1'illustre la figure 7, 1'augmentation serait comprise entre 1,3 et $1,7^{\circ} \mathrm{C}$, selon le scénario SRES B1, et entre 2,3 et $3{ }^{\circ} \mathrm{C}$, selon le scénario SRES A2.

\section{Évolution des températures maximales par rapport au seuil de $32{ }^{\circ} \mathrm{C}$}

Il a été montré que le nombre de jours où la température maximale dépasse $32{ }^{\circ} \mathrm{C}$ est en relation avec le risque de dengue, une maladie épidémique qui se manifeste certaines années en Nouvelle-Calédonie. Par exemple, lors de la saison 2008/2009, 8500 cas de dengue se sont déclarés sur une population de 250000 habitants. Comme pour le chikungunya ou le paludisme, l'épidémie se transmet par les moustiques dont le développement dépend de la température (Focks et al., 2000). Pour la prévision du risque de dengue, il faudrait surveiller, en particulier, si cette température est dépassée pendant 12 jours au cours de la période de janvier à mars (Descloux et al., 2011). Cependant, il faut bien noter que la température n'est pas le seul paramètre jouant un rôle dans l'occurrence d'épidémies.

Tableau 2 - Nombre de jours pendant lesquels la température maximale dépasse $32{ }^{\circ} \mathrm{C}$ pendant la période de janvier-février-mars, à Nouméa. Les cases sont colorées quand l'indice est supérieur au seuil de 12 jours. II s'agit des indices pour les 6 modèles, c'est pourquoi on a autant d'années (174 $=29 \times 6$ et $114=19 \times 6)$.

\begin{tabular}{llcl} 
& & $\begin{array}{c}\text { Indice moyen } \\
\text { (en jours) }\end{array}$ & $\begin{array}{l}\text { Pourcentage d'années pendant lesquelles } \\
\text { l'indice est supérieur à 12 jours }\end{array}$ \\
\hline $1971-1999$ & Observations & 7 & $17 \%$ (5 années sur 29) \\
\hline $1971-1999$ & $20 c 3 m$ & 9 & $29 \%$ (50 années sur 174) \\
\hline \multirow{2}{*}{$2046-2064$} & SRES B1 & 16 & $47 \%$ (54 années sur 114) \\
\cline { 2 - 4 } $2081-2099$ & SRES A2 & 21 & $66 \%$ (75 années sur 114) \\
\hline & SRES B1 & 21 & $73 \%$ (83 années sur 114) \\
\hline
\end{tabular}

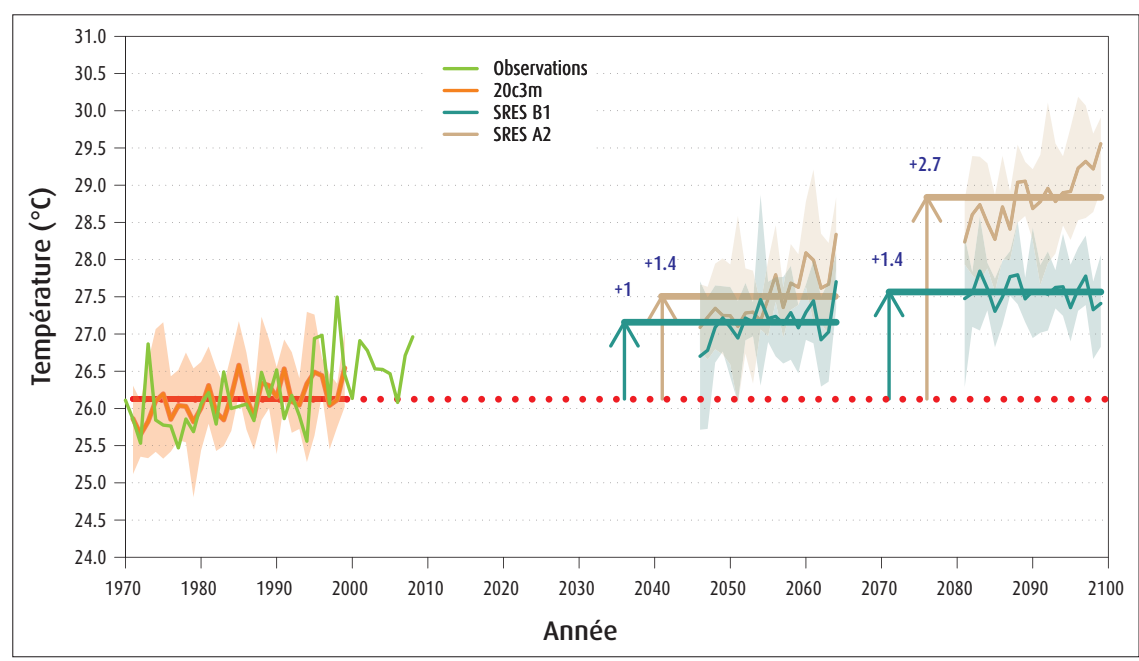

$\Delta$ Figure 7 - Variations temporelles de la température maximale annuelle, selon les observations et les projections, à Nouméa. Pour chaque scénario, le trait gras représente la moyenne des six modèles et les bandes ombrées représentent l'intervalle dans lequel se trouvent les valeurs des six modèles pris séparément.

₹Figure 8 - Variations temporelles des précipitations annuelles, selon les observations et les projections, à Nouméa. Voir la légende de la figure 7 . Rappel : $1 \mathrm{~mm}=1 \mathrm{~L} / \mathrm{m}^{2}$.

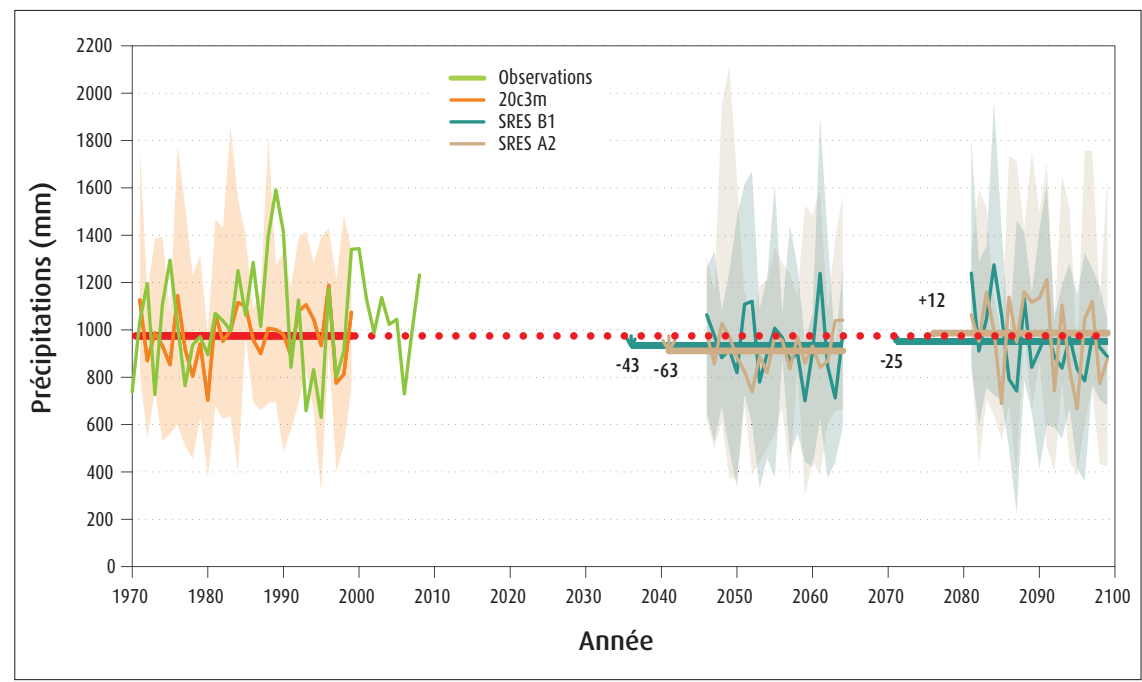

Comme le montre le tableau 2, le seuil de 12 jours pendant la période de janvier à mars serait largement dépassé à la fin du XXI ${ }^{\mathrm{e}}$ siècle, pour plus de $95 \%$ des années, selon le scénario SRES A2, et pour plus de $50 \%$ des années, selon le scénario SRES B1. Ceci est vrai quelle que soit la station sauf à Ouanaham où les valeurs sont un peu plus faibles. Si l'on compare la

simulation 20c3m aux observations, les modèles donnent un indice un peu trop fort à la fin du $\mathrm{xx}^{\mathrm{e}}$ siècle, mais l'augmentation est nette au cours du $\mathrm{XXI}^{\mathrm{e}}$ siècle.

Les conditions de températures du futur entraîneraient donc une augmentation de la fréquence des années météorologiquement favorables à l'épidémie de dengue. Comme il y aurait un allongement de la saison chaude, un allongement de la saison à risque pour cette maladie est à craindre.

\section{Évolution des précipitations}

En ce qui concerne les précipitations, il apparaît, sur la figure 8, que les variations des cumuls moyens de précipitations restent faibles en comparaison des fluctuations interannuelles. Il convient toutefois de rester prudent sur ce point car le choix des modèles joue un rôle 
important pour les précipitations, contrairement aux températures. Selon certains modèles, les variations sont positives alors que pour d'autres, elles sont négatives. En effectuant un test de Student, pour chaque modèle et pour chaque station, entre la simulation de contrôle $20 \mathrm{c} 3 \mathrm{~m}$ et chaque projection, il n'apparaît pas de variation significative des cumuls annuels de précipitations au niveau $5 \%$, sauf pour un modèle en 3 stations.

La répartition des précipitations au long de l'année pourrait toutefois changer. De la période actuelle à la fin du $\mathrm{XXI}^{\mathrm{e}}$ siècle, les précipitations diminueraient d'avril à juillet et elles augmenteraient de novembre à mars de manière significative au niveau $5 \%$, selon au moins deux modèles sur 6 , quelle que soit la station considérée. Cela rejoint l'analyse de Brown et al. (2011b) qui ont étudié les simulations des modèles du GIEC pour la fin du $\mathrm{XXI}^{\mathrm{e}}$ siècle, à grande échelle, dans la région Pacifique : « selon le scénario SRES A2, de décembre à février, il y a une petite hausse des précipitations dans la ZCPS, avec une influence potentielle mais incertaine sur la Nouvelle-Calédonie. De juin à août, la ZCPS des modèles tend à être trop zonale et à se déplacer légèrement vers le nord, mais sans grand changement des précipitations sur la NouvelleCalédonie. »

\section{À propos d'un éventuel risque de sécheresse}

Actuellement, la période la plus sensible du point de vue du risque de sécheresse se situe de juillet à octobre. Ce sont en effet les précipitations durant cette période qui conditionnent la sévérité de l'étiage (Frysou, 2008) car les dépressions des moyennes latitudes se font rares et la ZCPS, porteuse d'humidité, est encore trop au nord. Prenons, par exemple, le changement relatif de précipitations totales moyennes de juillet à octobre, selon SRES A2, entre 2081 et 2099 par rapport à 20c3m entre 1971 et 1999. À Bourail, Koumac, Touho et Ouanaham, les incertitudes restent fortes sur l'évolution des précipitations à cause de fortes divergences entre les 6 modèles. Les projections varient entre une diminution de précipitations de $40 \%$ et une augmentation équivalente, suivant le modèle considéré. Brown note également que « les changements les plus importants apparaissent en fait près de l'équateur et dans les zones de convergence ». La Nouvelle-Calédonie se trouve plus ou moins influencée par la ZCPS selon le positionnement de celle-ci par les modèles. On retiendra donc que les projections sont sujettes à une forte incertitude, liée à une représentation limitée de la ZCPS par les modèles du GIEC.

\section{Conclusion}

Avant d'analyser les séries de températures et de précipitations observées, il a été nécessaire de les homogénéiser, c'est-à-dire de corriger les éventuelles ruptures artificielles. Au cours des 40 dernières années, les moyennes annuelles des températures minimales et maximales quotidiennes se sont accrues respectivement de $0,3{ }^{\circ} \mathrm{C}$ et $0,2{ }^{\circ} \mathrm{C}$ par décennie, alors que les cumuls annuels de précipitations ne présentent pas de tendance significative, étant données les incertitudes. Au pas de temps quotidien, on décèle que le nombre annuel de jours pluvieux aurait augmenté au cours des 5 dernières décennies. De plus, les épisodes de pluies substantielles entre juin et août ont significativement diminué, en intensité et en nombre, sur la côte est et à Lifou. D'après les simulations mises en exergue dans le dernier rapport du GIEC (Hegerl et al., 2007), il est très probable que la cause principale du réchauffement du climat calédonien soit l'augmentation globale de la concentration des gaz à effet de serre dans l'atmosphère. En revanche, les évolutions pluviométriques constatées sont difficilement attribuables et pourraient résulter de fluctuations internes du système climatique dans le Pacifique sud-ouest sur une période de plusieurs décennies.

Les séries homogénéisées de températures au pas de temps quotidien ont ensuite servi de référence pour l'étude du climat du futur. Les simulations climatiques produites par les modèles du GIEC n'ont pas une résolution suffisante pour conduire une étude d'impact à échelle fine sur la NouvelleCalédonie. Par conséquent, une régionalisation des données climatiques par la méthode statistique de descente d'échelle quantile-quantile a été réalisée.

Les principaux résultats sont les suivants :

- les températures minimales et maximales augmenteraient, pour toutes les stations, d'environ $1{ }^{\circ} \mathrm{C}$, selon le scénario d'émissions limitées (SRES B1), et de $1,4{ }^{\circ} \mathrm{C}$, selon le scénario d'émissions persistantes (SRES A2), de la période actuelle à la période
2046-2064. Cette augmentation serait d'environ $1,5^{\circ} \mathrm{C}$, selon le scénario SRES B1, et de $2,7^{\circ} \mathrm{C}$, selon le scénario SRES A2, de la période actuelle à la période 2081-2099;

- la saison chaude, telle qu'on la connaît actuellement, durerait deux ou trois mois de plus (respectivement selon SRES B1 ou SRES A2) à la fin du XXI ${ }^{\mathrm{e}}$ siècle ;

- les conditions de températures du futur entraîneraient une augmentation de la fréquence des années climatiquement favorables à des épidémies de dengue ;

- les précipitations totales annuelles ne montrent pas de tendance significative ;

- la distribution des précipitations au long de l'année pourrait changer, avec une diminution d'avril à juillet et une augmentation de novembre à mars, suggérées par certains modèles, mais de juillet à octobre (période critique pour la sévérité de l'étiage) les désaccords entre les 6 modèles sont trop forts pour pouvoir conclure.

Cette étude confirme la présence d'un risque pour la Nouvelle-Calédonie lié au réchauffement climatique, d'où la nécessité d'approfondir notre connaissance du climat du futur. On peut regretter que les simulations utilisées aient toutes une représentation grossière de la Nouvelle-Calédonie, sans point de terre dans la grille du modèle atmosphérique. La méthode quantilequantile gagnerait à être appliquée à des températures simulées sur des points de terre. Ce petit territoire échappe aux modèles globaux de climat mais il pourrait être mieux représenté par des modèles numériques locaux imbriqués dans un modèle global. D'autre part, l'évolution des précipitations reste difficile à évaluer car les divergences entre les modèles sont fortes, ce qui pourrait être amélioré par une meilleure simulation des phénomènes, notamment la zone de convergence du Pacifique sud, à l'échelle de l'océan Pacifique.

\section{Remerciements}

Nous souhaitons rendre hommage aux observateurs volontaires et aux agents du Service météorologique de la Nouvelle-Calédonie qui ont œuvré pour rendre la base de données climatologiques aussi fiable et étoffée. 


\section{Bibliographie}

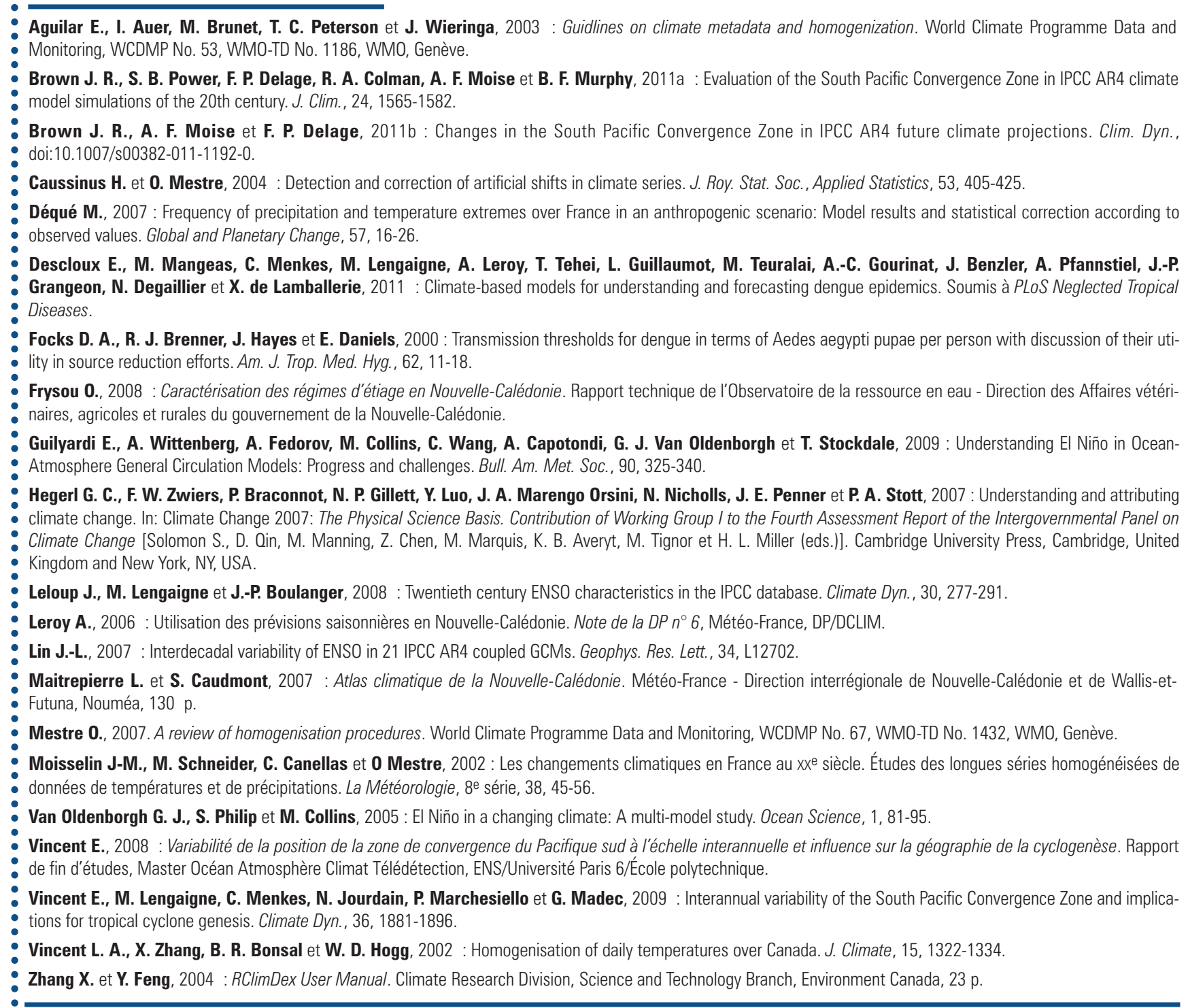

